Conventional Publication Bias Correction Methods

\author{
Julia Haaf ${ }^{1}$ \\ ${ }^{1}$ University of Missouri
}

Author Note

Roughly 3700 words.

Correspondence concerning this article should be addressed to Julia Haaf. E-mail: jhaaf@mail.missouri.edu 


\section{Conventional Publication Bias Correction Methods}

"Journals are filled with the 5\% of the studies that show Type I errors, while the file drawers are filled with the $95 \%$ of the studies that show non-significant results." (Rosenthal, 1979)

\section{Introduction}

In psychology, the number of studies investigating a certain effect is often taken as direct evidence fot the effect. For example, there is an overwhelming number of studies using the Stroop task, providing evidence for the validity of the Stroop effect (MacLeod, 1991; e.g. Stroop, 1935). The reason why this simple counting rule works is because most studies in the literature show and effect rather than its absence. Three reasons for this phenomenon of positive evidence in the literature immediately come to mind. First, an effect is a strong effect if it can be found in almost any paradigm that is used to study the effect. Indeed, this seems to be the case for the Stroop effect. MacLeod (1991) even argues that most likely everyone who can read exhibits a Stroop effect. Second, editors and publishers may prefer significant results because they are catchier. Third, authors may see non-significant results as a failure of the experimental procedure rather than negative evidence for an effect. Authors may be right with that notion because non-significant results in the common frequentist framework are due to the failure to reject the null and cannot be interpreted as evidence for or against anything.

The latter two explanations for the phenomenon of positive evidence can be summarized as the usage of publication rules based on the outcome of a study (Sterling, 1959). This procedure most evidently lead to gaps in the psychological literature. Almost everybody who tried to publish in psychology knows how hard it is to publish studies with less than catchy results. This outcome-based publication rule was introduced as the file drawer problem (Rosenthal, 1979) and is now commonly called publication bias. Both file drawer and publication bias refer the phenomenon that null results (i.e. studies with 
non-significant results) rarely get published. Publication bias is problematic when the scientific literature is reviewed. An example of a reiew method is meta-analysis. Here, evidence for an effect or a phenomenon is analyzed on the basis of a comprehensive gathering of (mainly published) studies. Obviously, if only significant studies get published, the results of such an analysis may be extremely distorted. How to detect and correct for publication bias in meta-analysis is therefore timely and topical.

\section{Detecting Publication Bias}

A.

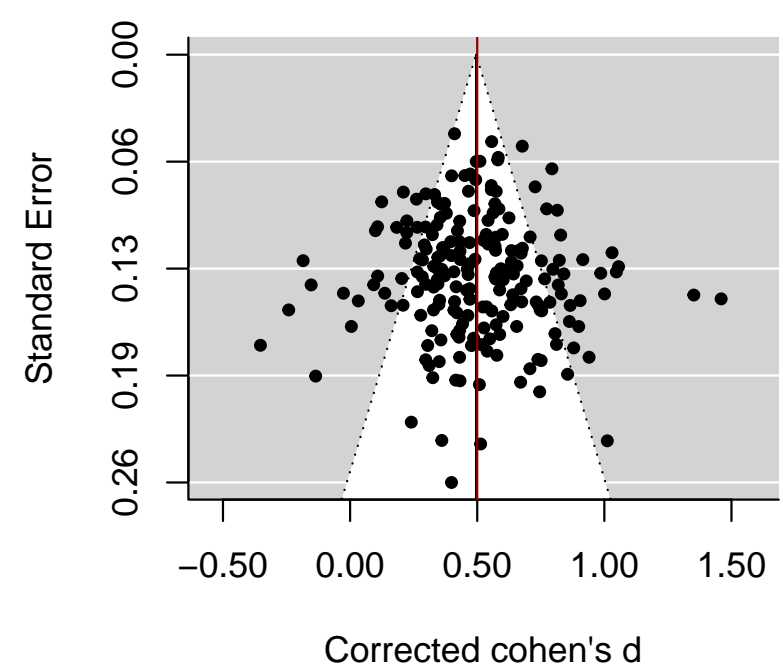

B.

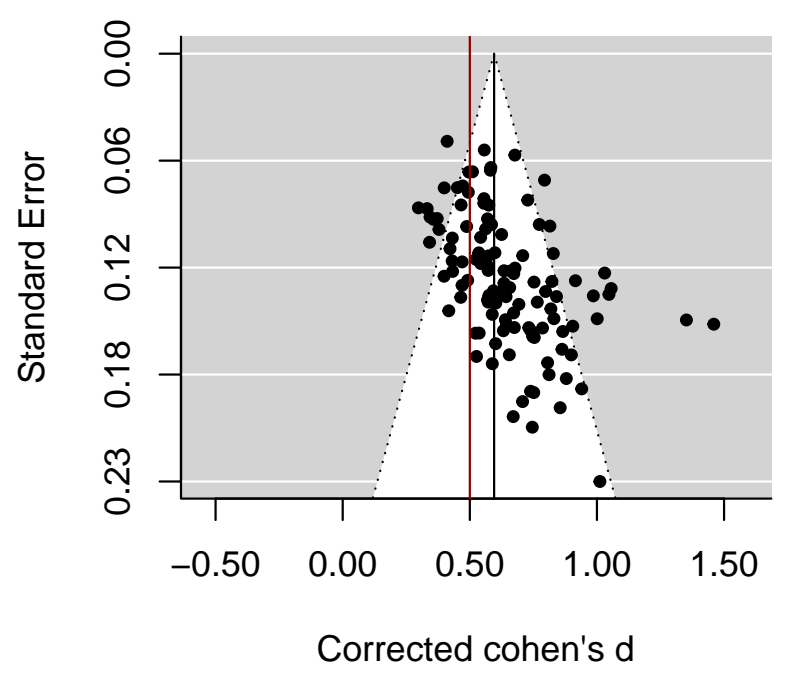

Figure 1. Funnel plots for simulated data for A. all 200 simulated data sets and B. the data sets with $p<.05$ that can be assumed to enter the literature.

A graphical way to detect publication bias in a meta-analytic set is a funnel plot (Egger, 1997).The idea of these plots is that the estimated effect size is a function of the sample size. For small samples, most variability in effect size measures is expected. For larger samples, the observed effect sizes should converge to the true meta-analytical effect size. Ideally, one could extrapolate the true effect size by drawing a funnel around the observed data points in the plot. Figure 1 shows two funnel plots for a simulated data set. The data set consists of 200 studies with varying sample size. Samples are drawn from a 
normal distribution with true effect size of 0.5. In the figure, the x-axis depicts the observed effect size, the y-axis depicts the standard error, which in turn is a function of sample size. The black vertical line shows the estimated overall effect size. The white funnels are pseudo 95\% confidence interval regions. Panel A shows the funnel plot for all studies in the set. In a meta-analytical set without publication bias, the observed effects should vary symmetrically around the true effect size - here depicted by the red line - and they should converge to the true effect size as the sample size increases. This is the case with this simulated data set. Panel B shows the same simulated set, but it is censored so that only effects significant at an $\alpha=.05$ level are used. This censoring represents an extreme publication bias, where only significant studies are published. A meta-analysis of published findings for a paradigm would therefore only include these studies. For the simulated set here, 112 of the 200 studies yield a significant result. The resulting funnel plot is asymmetric with the majority of observed effect sizes being larger than the true effect size. The resulting effect size estimate is 0.60 , clearly larger than the true effect size of .5.

Simulation studies like the one conducted here are commonly taken as evidence that asymmetric funnel plots are due to publication bias in the meta-analytic set. If many studies with small effect sizes and/or non-significant results are omitted, the resulting funnel plot essentially misses one of the tails of the effect size distribution. Recently, this funnel plot inspection approach has been criticized. For example, Sterne et al. (2011) note that funnel plots can be asymmetric due to correlations between an effect and the standard error and not due to publication bias. Considering this critique, it seems unclear whether researchers can easily detect publication bias - or the extent of it - with common methods such as funnel plot inspection. Yet, it seems highly plausible that there is publication bias in many areas in psychological science. The main question then is how to correct for such a bias. 


\section{Correcting for Publication Bias}

One of the first proposals on how to deal with publication bias was provided by Rosenthal (1979). Rosenthal conducted a calculation to assess the severity of publication bias. The basic idea is that one can calculate an effect size measure from the studies published and assess how many studies must be hidden in file drawers to outweigh the published results. For this calculation Rosenthal uses $z$-scores associated with the published $p$-values and the number of published experiments, $k$. The $z$-scores from all published

experiments can be combined to one joint $Z_{c}$, where $Z_{c}=\frac{\sum z_{k}}{\sqrt{k}}$. This combined $Z_{c}$ is significant when $Z_{c}>1.64$, given an $\alpha$ level of .05 and a directional hypothesis. For any given number of experiments and their combined $Z_{c}$, we can calculate the number of file drawer experiments, $X$, that are needed to reduce $Z_{c}$ to a non-significant value. For example, if the combined $Z_{c}=5$ and the number of published experiments is $k=100$, then number of file drawer experiments needed is $X=824$. Rosenthal argues that this number is fairly high, maybe too high to be realistic. He concludes therefore that publication bias does not affect the judgement of whether an effect in the literature is valid or not.

Since the article by Rosenthal (1979), the debate about the impact of publication bias has shifted from the question whether a certain effect exists to how big a certain effect is. This debate is most prominent in the meta-analytical literature, where the outspoken goal is to estimate population effect sizes for various psychological effects. Publication bias most certainly has an impact on the estimated effect size of an effect: If only significant results are published, smaller observed effects may be omitted in the literature and therefore cannot be incorporated in conventional estimation methods.

Here, I want to summarize current means to counteract publication bias in effect size estimation in meta-analyses. The most prominent methods in the meta-analytic literature are trim and fill (Duval \& Tweedie, 2000), p-Curve (Simonsohn, Nelson, \& Simmons, 2014), meta-regression estimation (i.e. PET/PEESE, Stanley \& Doucouliagos, 2014), and selection methods (Hedges, 1984; Iyengar \& Greenhouse, 1988). I summarize these methods in turn 
and discuss advantages and disadvantages subsequently.

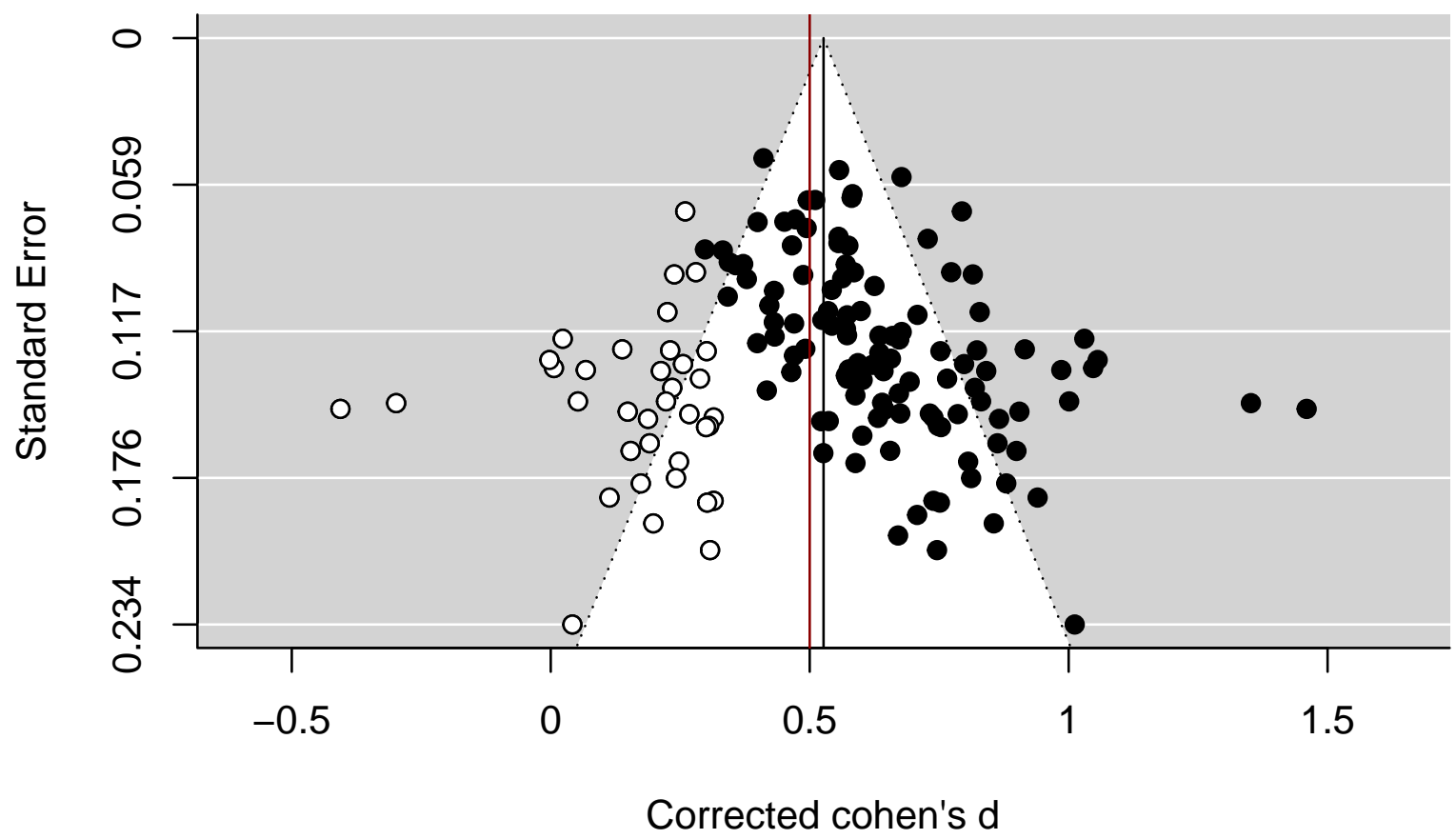

Figure 2. Funnel plot illustrating the trim-and-fill method. Filled points denote original data set, the unfilled points denote added data points from the trim-and-fill method. The resulting effect size estimate is close to the true effect size denoted by the red line.

Trim and Fill. Trim and fill (Duval \& Tweedie, 2000) is a common method for publication bias correction. The method uses a measure of asymmetry in funnel plots to first detect the extent of publication bias and then to correct for it by trimming data points that increase asymmetry and filling in data points to counterbalance until symmetry is reached. The resulting data set can then be used for common meta-analytical analyses. Figure 2 shows the application of the trim and fill method to the simulated data set. The resulting estimate is 0.53 , which is closer to the true effect size of .5 than the estimate of the untreated data set. Yet, the true effect size is still overestimated.

$p$-Curve and $p$-Uniform. The $p$-curve method was introduced by Simonsohn et al. (2014) to test whether there was a true underlying effect and to estimate the average effect size. A $p$-curve is the distribution of (significant) p-values in a meta-analytical set. The 


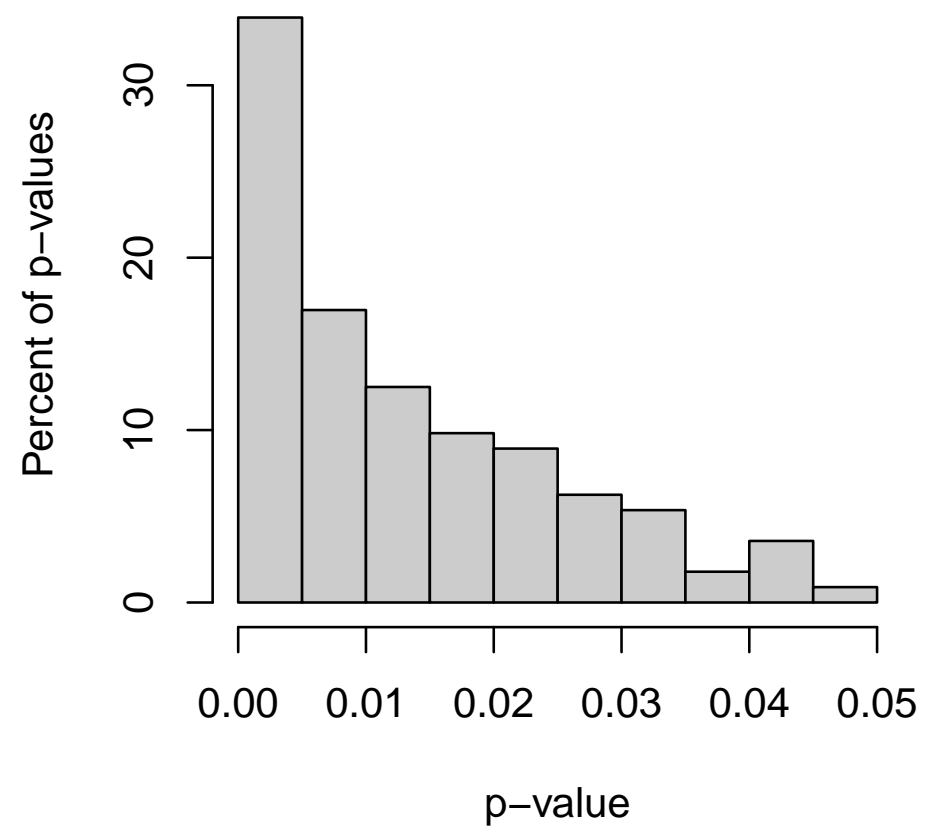

Figure 3. p-curve for the censored meta-analytical set. The curve is clearly right-skewed as expected when an effect actually exists.

rationale is as follows: If the underlying true effect size is zero, then the $p$-values should be distributed as a uniform, $p \sim \operatorname{Uniform}(0,1)$. The reason for this is trivial: Under the null hypothesis, $p<.10$, for example, will occur $10 \%$ or the time, $p<.09$ will occur $9 \%$ of the time, and $p<.08$ will occur $8 \%$ of the time. Dividing $p$-values in bins of .01 then leads to $1 \%$ of $p$-values between .10 and .09 , and $1 \%$ between .09 and .08, and so on. If we are only concerned with significant values, $p<.05$, these should also be uniform, $p \sim \operatorname{Uniform}(0, .05)$.

In contrast, if the underlying true effect size is larger than zero, the distribution of significant $p$-values should be right-skewed, i.e. significant results with $0<p<.01$ should be more likely than $.01<p<.02$ and so on. An example of such a distribution is shown in Figure 3. The figure shows a histogram of $p$-values from the simulated example. Only $p<.05$ are shown, coming from the 112 studies that yield a significant result. The resulting $p$-curve is clearly right-skewed, indicating a true effect size greater that zero. In this case, $\delta=.5$, and the $p$-curve seems diagnostic of that.

Similar to $p$-curve, the $p$-uniform method is also concerned with the distribution of 
statistically significant $p$-values (Assen, Aert, \& Wicherts, 2015). Note that the $p$-uniform method, unlike the $p$-curve method, focuses on the distribution of $p$-values conditional on the population effect size. Two such conditional distributions of $p$-values are considered: First, the distribution of $p_{\delta=0}$-values is the distribution of $p$ s conditional on $\delta=0$, where $\delta$ is the population effect size. If the null hypothesis, $\delta=0$ is true, this distribution is $p_{\delta=0} \sim \operatorname{Uniform}(0,1) .{ }^{1}$ Second, the distribution of $p_{\delta=\delta_{0}}$, is the distribution of $p$ s under the alternative hypothesis. If $\delta=\delta_{0}, p_{\delta=\delta_{0}} \sim \operatorname{Uniform}(0,1)$.

Both methods allow to estimate the population effect size, $\delta$, and to test the null hypothesis that $\delta=0$. $p$-uniform additionally provides confidence intervals. A disadvantage of both methods is that they are only applicable to fixed-effect meta-analyses, not to random-effects meta-analysis that are becoming more popular.

Meta-Regression Approaches PET, PEESE, and PET-PEESE. The meta-regression approaches PET, PEESE and PET-PEESE utilize the relationship between standard error and effect size to fit a regression model. For PET, the precision-effect test, a linear regression is fit to the effect size with the standard error as predictor (Stanley \& Doucouliagos, 2014). If the intercept is significantly different from zero, it can be used as population effect size estimate because it corresponds to the case where $S E=0$. Figure 4 shows the regression line for the PET method and the corresponding effect size estimate (blue line and circle). The intercept is significantly different from zero, but it greatly underestimates the true effect size, denoted by the red line. Stanley and Doucouliagos (2007) note that this underestimation may be due to a nonlinear relationship between the standard error and the effect size, at least when there is a substantial overall effect size.

An alternative approach, the precision-effect estimate with SE - short PEESE therefore employs a quadratic relationship between standard error and effect size (Stanley \& Doucouliagos, 2014). Other than the quadratic function, the approach is identical to PET.

${ }^{1}$ The domain of the distribution, $[0,1]$, refers to transformed $p$-values. The transformation is described by Assen et al. (2015) 


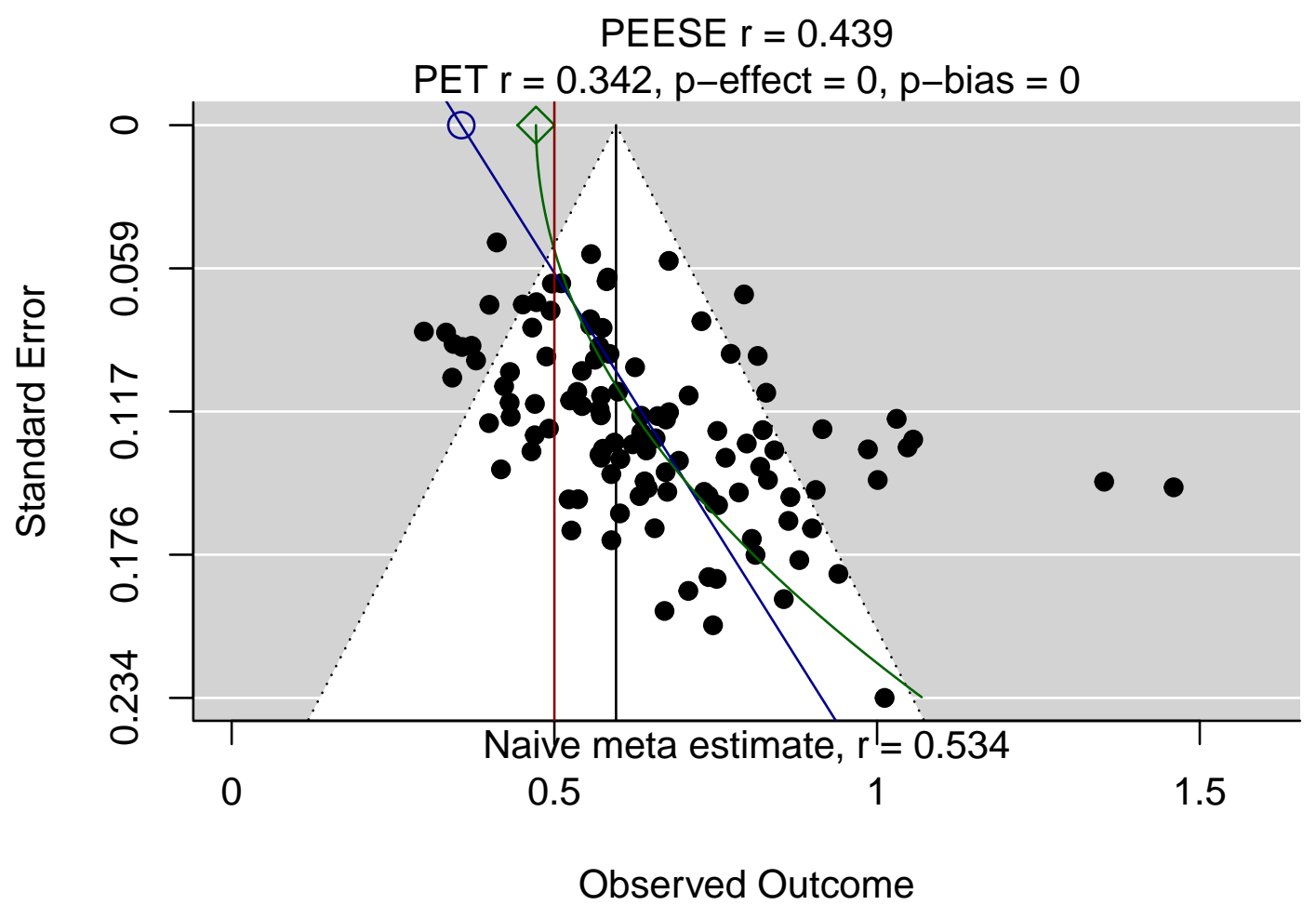

Figure 4. PET-PEESE analysis for the censored meta-analytical set. Since the estimate from the PET analysis is significant, the PEESE estimate is taken as final estimate. Both methods underestimate the true effect size for this case, but the PEESE estimate is very close.

The regression line and estimate for the simulation example are also shown in Figure 4 (green line and diamond). The estimate is much closer to the true effect size, but it still underestimates it.

But if PEESE performs better in estimation, why even use PET? Stanley and Doucouliagos (2014) showed in simulation studies that PET performs better at identifying true zero-effects. PEESE, on the other hand leads to better estimates when the true effect size is non-zero. The authors therefore recommend using a mix of both methods, called PET-PEESE. First, the PET method is used to decide whether there is a significant non-zero effect size in the population. If the PET analysis shows a significant result, PEESE is then used to estimate the population effect size. In the case of the current simulation study, PET yields a significant result, so the PEESE estimate would be used as population estimate. 
Selection Models. Selection models were first introduced by Hedges (1984). These models explicitly model the process of publication bias, or non-random sampling of studies. The underlying assumption in the original paper was the strict publication bias that only significant results were published. This assumption was later relaxed (Iyengar \& Greenhouse, 1988). The selection models now commonly assume that significant results are always published and non-significant results are published with some probability $p$. This parameter $p$, together with the population effect size parameter $\mu$ and the variability of effects, $\tau^{2}$, can be estimated in a random-effects model using maximum likelihood methods.

\section{Comparison}

The described methods for correcting publication bias - trim and fill, $p$-curve $/ p$-uniform, PET/PEESE, and selection models - can be classified into three different approaches:

- trim and fill and PET/PEESE are based on the same relationship between the standard error and the effect size of a study that is also used to create funnel plots. Even though trim and fill tends to overestimate and PET/PEESE tends to underestimate the population effect size, the methods are based on the same assumption that the function and symmetry of this relationship is meaningful. I call these methods $S E$-methods.

- $p$-curve and $p$-uniform are based on the assumption that the distribution of $p$-values can be used to spot publication bias and correct for it. I call these methods $p$-distribution-methods.

- Selection models are an extension of random-effects models commonly used in meta-analysis. These models employ varying but detailed assumptions about the publication process, namely if and how likely non-significant results are published. I call these selection-methods. 
There are various ways these methods can be compared to each other. For example, one can question the assumptions of $S E$-methods and selection methods. In the literature, the most common comparison seems to be the performance of the methods in simulation studies.

Simulation Studies. Simulation studies are commonly used to compare the above methods, because they are the only way to compare set true parameter values with parameter estimates. In simulation studies for publication bias correction methods, various parameters such as sample size, true effect size, true effect variability and statistical power are manipulated to show how the methods perform in varying settings. Here, I summarize results from two simulation studies conducted by McShane, Böckenholt, and Hansen (2016) and Carter, Schönbrodt, Hilgard, and Gervais (2017). I then argue that these simulation studies may be flawed and not the ideal basis of a decision for any one of the methods.

McShane et al. (2016) conducted a simulation study to compare selection methods with $p$-distribution methods. They varied I. the true effect size $\delta=.1, .3, .5$; II. the number of published studies, $k=20,40,60,80,100$; III. the probability that non-significant studies are published, $q=0, .1, .25$; IV the variability of true effects, $\tau=0, .2$. Overall, the selection models perform better than the $p$-distribution methods, especially when the probability of non-significant studies being published is non-zero, when the true effect size is small, and when true effect sizes are heterogeneous.

Carter et al. (2017) attempted a simulation study that reflects typical parameters of a meta-analytical set in psychology. The authors state that heterogeneity of effects is fairly large and effect sizes are fairly small in psychology. They also attempted to include questionable research practices (QRPs) in their simulation, an issue commonly assumed in psychological science (John, Loewenstein, \& Prelec, 2012). The authors manipulated several experimental factors: I. the true effect size $\delta=0, .2, .5, .8$; II. the variability of true effects, $\tau=0, .2, .4 ;$ III. the number of published studies, $k=10,30,60,100 ;$ IV . proportion of studies produced under publication bias $p_{\text {bias }}=0, .6, .9 ; \mathrm{V}$. Amount of QRPs (none, medium and high). The crossing of all these factors results in 432 conditions, and the authors 
simulated 1000 meta-analyses for each condition. They applied all the above introduced methods to these meta-analyses and compared 1. the accuracy of significance testing decisions, i.e. whether the methods yielded a significant non-zero estimate when $\delta \neq 0$, and a non-significant result when $\delta=0$, and 2. the precision of the estimated effect size, $\hat{\delta}$.

Overall, the selection method yielded quite conservative testing decisions with relatively low power, while trim and fill or no-correction random-effects meta-analysis resulted in close to $100 \%$ false positives when $\delta=0$ and $p_{\text {bias }}>0$. Trim and fill, no-correction, and $p$-distribution methods lead to an overestimation of $\hat{\delta}$ when variability of true effect sizes was large. Conversely, PET/PEESE methods lead to a vast underestimation of $\hat{\delta}$. Selection models performed best under these difficult conditions, but the true effect size was still underestimated.

Both simulation studies conclude a (slight) preference for the selection methods. Yet, these results should be treated with caution. McShane et al. (2016), for example, tweaked the fitted selection model as a function of true values of their simulation. When $\tau=0$ and $q=0$, they used the original model from Hedges (1984), a fixed-effects model that assumes that no non-significant studies were published. When $\tau=0$ and $q>0$, they used the model introduced by Iyengar and Greenhouse (1988), a fixed-effect model that assumes that some non-significant studies were published. When $\tau>0$ and $q>0$, they extended this model to a random-effects model. This approach is not very useful. Obviously, if one of the models has similar features than the ones used to simulate data, the model will most likely be preferred. Similarly, Carter et al. (2017) emphasize the more difficult simulation conditions, that are best represented by the selection methods. For obvious reasons, this methods performs best under the circumstances it assumes.

Generally speaking, simulation studies are a function of the assumptions the simulations are based on. The circumstances under which publication bias occurs, or even under which studies are selected for a meta-analytical set, are most likely more complicated than what a simulation study can accomplish. Carter et al. (2017) argue that their 
simulations are closer to the reality in psychological science than previous studies, but they are still based on assumptions of censoring processes, true variability and true effect sizes. We do not know how close these assumptions are to reality. Moreover, these assumptions are most likely closer to the principles of the correction methods than to reality.

Bayesian Approach. Guan and Vandekerckhove (2016) introduced a promising Bayesian approach that extends the selection-method approach. They propose several censoring processes, for example the extreme-bias model where only significant studies are published, the constant-bias model where non-significant studies are published with probability $\pi$, regardless of their $p$-value, and the exponential-bias model, where non-significant studies are published with a probability that is inverse proportional to its $p$-value. The authors propose a Bayesian model averaging approach, where these different censoring models can be applied to any common meta-analysis, such as random-effects or fixed-effect meta-analyses. Using Bayes' rule, we can then marginalize over these different censoring models, resulting in an aggregated posterior estimate for the true effect size.

This apporach avoids too strong assumptions about the publication bias process by averaging over different options. So far, the approach does well in simulation (Guan \& Vandekerckhove, 2016). Yet, to my knowledge, it has not been compared to other approaches. Even so, performance in simulation may not be the best tool to evaluate the quality of any of the correction methods including the one by Guan and Vandekerckhove.

\section{Conclusion}

It is safe to conclude that publication bias is indeed an issue for the estimation of population effect sizes in a meta-analytical framework. Many correction methods have been

proposed with varying advantages and disadvantages. Comparison between these methods is difficult, and it is done mostly in simulation settings. Yet, these simulation studies are problematic, because they mirror the assumptions underlying the correction methods they aim to compare. New ways to compare correction methods are needed. One approach may 
be to compile a meta-analytical dataset for a psychological paradigm, and to also run a study in many-labs fashion, where different labs run the same experiment (e.g. Ebersole et al., 2016). Different correction methods can be applied to the meta-analytical set, and the resulting effect size estimates can be compared to the overall estimate from the many-labs study. The many-labs set could also be used to simulate QRPs: The participating researchers may be instructed to p-hack and manipulate their own study in any fashion known to them. The QRP-ed set could then be compared to the raw dataset, and this comparison could yield new insights to the effect of common QRPs.

The key result of a review on publication bias is that meta-analysis may be overly trusted. While it is important to aggregate results from smaller studies to obtain more robust results, a meta-analysis of the literature may not be the ideal way. Alternatives are large-scale many-labs or replication projects, where transparency and openness guidelines naturally limit publication bias and QRPs. 


\section{References}

Assen, M. A. L. M. van, Aert, R. C. M. van, \& Wicherts, J. M. (2015). Meta-analysis using effect size distributions of only statistically significant studies. Psychological Methods, 20, 293-309. Retrieved from http://psycnet.apa.org/doi/10.1037/met0000025

Carter, E. C., Schönbrodt, F. D., Hilgard, J., \& Gervais, W. M. (2017). Correcting for bias in psychology: A comparison of meta-analytic methods. Retrieved from https://osf.io/preprints/psyarxiv/9h3nu/

Duval, S., \& Tweedie, R. (2000). Trim and fill: A simple funnel-plot-based method of testing and adjusting for publication bias in meta-analysis. Biometrics, 56, 455-463. Retrieved from 10.1111/j.0006-341X.2000.00455.x

Ebersole, C. R., Atherton, O. E., Belanger, A. L., Skulborstad, H. M., Allen, J. M., Banks, J. B., ... Nosek, B. A. (2016). Many labs 3: Evaluating participant pool quality across the academic semester via replication. Journal of Experimental Social Psychology, 67, 68-82. Retrieved from http://ezid.cdlib.org/id/doi:10.17605/OSF.IO/QGJM5

Egger, M. (1997). Bias in meta-analysis detected by a simple, graphical test. British Medical Journal, 315, 629-634. Retrieved from DOI: 10.1136/bmj.315.7109.629

Guan, M., \& Vandekerckhove, J. (2016). A Bayesian approach to mitigation of publication bias. Psychonomic Bulletin and Review, 23(1), 74-86. Retrieved from http://www.cidlab.com/prints/guan2015bayesian.pdf

Hedges, L. V. (1984). Estimation of effect size under nonrandom sampling: The effects of censoring studies yielding statistically insignificant mean differences. Journal of Educational Statistics, 9(1), 61-85.

Iyengar, S., \& Greenhouse, J. B. (1988). Selection models and the file drawer problem. Statistical Science, 109-117.

John, L. K., Loewenstein, G., \& Prelec, D. (2012). Measuring the prevalence of questionable research practices with incentives for truth telling. Psychological Science, 23(5), 
524-532. Retrieved from http://pss.sagepub.com/content/23/5/524.abstract

MacLeod, C. (1991). Half a century of research on the Stroop effect: An integrative review. Psychological Bulletin, 109, 163-203.

McShane, B. B., Böckenholt, U., \& Hansen, K. T. (2016). Adjusting for publication bias in meta-analysis: An evaluation of selection methods and some cautionary notes. Perspectives on Psychological Science, 11(5), 730-749.

Rosenthal, R. (1979). The file drawer problem and tolerance for null results. Psychological Bulletin, $86(3)$, 638-641.

Simonsohn, U., Nelson, L. D., \& Simmons, J. P. (2014). P-curve: A key to the file-drawer. Journal of Experimental Psychology: General, 143, 534-547. Retrieved from $10.1037 / \mathrm{a} 0033242$

Stanley, T. D., \& Doucouliagos, H. (2007). Identifying and correcting publication selection bias in the efficiency-wage literature: Heckman meta-regression. Economics Series, 11. Retrieved from https://ideas.repec.org/p/dkn/econwp/eco_2007_11.html

Stanley, T. D., \& Doucouliagos, H. (2014). Meta-regression approximations to reduce publication selection bias. Research Synthesis Methods, 5(1),60-78. Retrieved from DOI: $10.1002 / \mathrm{jrsm} .1095$

Sterling, T. D. (1959). Publication decisions and their possible effects on inferences drawn from tests of significance - or vice versa. Journal of the American Statistical Association, 54, 30-34.

Sterne, J. A. C., Sutton, A. J., Ioannidis, J. P. A., Terrin, N., Jones, D. R., Lau, J., ... Deeks, J. J. and. (2011). Recommendations for examining and interpreting funnel plot asymmetry in meta-analyses of randomised controlled trials. BMJ, 343 . Retrieved from http://www.bmj.com/content/343/bmj.d4002

Stroop, J. R. (1935). Studies of interference in serial verbal reactions. Journal of Experimental Psychology, 18, 643-662. 